\title{
Le genre Entandrophragma (Meliaceae) : taxonomie et écologie d'arbres africains d'intérêt économique (synthèse bibliographique)
}

\author{
Emmanuel Kasongo Yakusu ${ }^{(1,2,3)}$, Franck S. Monthe ${ }^{(4)}$, Nils Bourland ${ }^{(3,5,6)}$, \\ Olivier J. Hardy ${ }^{(4)}$, Dominique Louppe ${ }^{(7)}$, Félicien Bola Mbele Lokanda ${ }^{(8)}$, \\ Wannes Hubau $^{(3)}$, Jean-Marie Kahindo Muhongya ${ }^{(8)}$, Jan Van Den Bulcke ${ }^{(2)}$, \\ Joris Van Acker ${ }^{(2)}$, Hans Beeckman ${ }^{(3)}$
}

(1) Université de Kisangani. Faculté de Gestion des Ressources Naturelles Renouvelables. B.P. 2012. Avenue Kitima, 3.

Kisangani (République Démocratique du Congo). E-mail : emmakasongo1@yahoo.fr

(2) Université de Gand. Laboratoire de Technologie du Bois. Coupure Links, 653. BE-9000 Gand (Belgique).

(3) Musée Royal de l'Afrique Centrale. Service de Biologie du Bois. Leuvensesteenweg, 13. BE-3080 Tervuren (Belgique).

(4) Université libre de Bruxelles. Faculté des Sciences. Évolution biologique et Écologie. CP 160/12. Avenue F. D. Roosevelt, 50. BE-1050 Bruxelles (Belgique).

(5) Centre de Recherche Forestière Internationale. Situ Gede Bogor Barat 16115 (Indonésie).

(6) Resources and Synergies Development. Noliktavu iela, 7. Dreilini, Stopinu novads. Riga 2130 (Lettonie).

(7) Centre de coopération Internationale en Recherche Agronomique pour le Développement (Cirad). Département forestier. Campus international de Baillarguet. TA 10/C 34398. FR-Montpellier Cedex 5 (France).

(8) Université de Kisangani. Faculté des Sciences. B.P. 2012. Avenue Kitima, 3. Kisangani (République Démocratique du Congo).

Reçu le 28 juin 2017, accepté le 23 janvier 2018, mis en ligne le 6 mars 2018.

Cet article est distribué suivant les termes et les conditions de la licence CC-BY (http://creativecommons.org/licenses/by/4.0/ deed.fr)

Introduction. Les espèces d'arbres du genre Entandrophragma font l'objet d'une attention toute particulière de la part des exploitants forestiers et des scientifiques depuis plusieurs décennies. Établir un bilan exhaustif actualisé des connaissances acquises sur leur taxonomie et leur écologie afin d'en dégager des pistes de nouvelles recherches est le but de cette synthèse bibliographique.

Littérature. Le genre Entandrophragma compte des espèces (10 à 12) exclusivement africaines et qui sont exploitées pour la qualité de leur bois. Ces espèces sont répandues dans la région guinéo-congolaise (6 espèces) et dans les régions zambézienne et afromontagnarde (5 espèces). Le genre est caractérisé par une évolution taxonomique qui s'est traduite par une importante synonymie des noms d'espèces (36 à 37). Des études écologiques un peu larges ont été réalisées sur quelques principales espèces commerciales (Entandrophragma angolense, Entandrophragma congoense, Entandrophragma candollei, Entandrophragma cylindricum, Entandrophragma palustre et Entandrophragma utile) dans leurs zones phytogéographiques comprises principalement dans la région guinéo-congolaise.

Conclusions. Les informations sur la taxonomie et l'écologie des espèces d'arbres d'Entandrophragma des forêts claires et des savanes sont insuffisantes comparativement à celles des forêts denses humides. Des travaux de recherches devraient être développés pour l'amélioration des connaissances taxonomiques, génétiques et la maitrise de leur écologie en vue de formuler des recommandations adéquates en matière de préservation et de gestion durable de ces espèces.

Mots-clés. Afrique, distribution géographique, écologie forestière, Entandrophragma, forêt tropicale, génétique des populations, taxonomie des essences forestières. 
Entandrophragma: taxonomy and ecology of a genus of African tree species with economic importance. A review

Introduction. Entandrophragma tree species have been the subject of special attention by foresters and scientists for several decades. The purpose of this bibliographic review is to examine the current knowledge acquired on the taxonomy and ecology of this genus in order to identify new research priorities.

Literature. The genus Entandrophragma has exclusively African species (10 to 12) that are exploited for the quality of their wood. These species are widespread in the Guineo-Congolese region (6 species) and in the Zambezian and Afromontane regions ( 5 species). The genus is characterized by a taxonomic evolution that has resulted in an important synonymy of species names (36 to 37). Ecological studies have been carried out on some major commercial species (Entandrophragma angolense, Entandrophragma congoense, Entandrophragma candollei, Entandrophragma cylindricum, Entandrophragma palustre and Entandrophragma utile) in their phytogeographic zones, mainly in the Guineo-Congolese region.

Conclusions. Taxonomy and ecology of Entandrophragma tree species typically occurring in woodlands and savannas have been insufficiently studied in comparison with Entandrophragma species occurring in rainforests. Research needs to be developed for the improvement of taxonomic, genetic and ecological knowledge, with a view to formulating appropriate recommendations for the conservation and sustainable management of these species.

Keywords. Africa, forest ecology, Entandrophragma, population genetic, natural range, taxonomy of tree species, tropical forests.

\section{INTRODUCTION}

Le genre Entandrophragma C.DC. comprend, selon les sources, 10 à 12 espèces d'arbres distribuées exclusivement en Afrique tropicale. Appartenant à la tribu des Swietenieae A.Juss. et à la famille des Meliaceae, il est apparenté aux genres Lovoa Harms, Khaya A.Juss, Pseudocedrela Harms (Lemmens, 2008) et Cedrela P.Browne (Koenen et al., 2015).

$\mathrm{Au}$ sein de la famille des Meliaceae, le genre Entandrophragma est le plus riche en espèces précieuses exploitées comme bois d'œuvre en Afrique (Tailfer, 1989). Au-delà de son importance économique, ce genre présente un intérêt scientifique, notamment sur le plan taxonomique, vu la multiplicité des genres auxquels ont été rattachées dans un premier temps les espèces d'Entandrophragma et le nombre des synonymies spécifiques (36-37) (Louis \& Fouarge, 1947 ; Hall, 2008 ; The Plant List, 2013).

L'insuffisance des informations sur l'écologie des essences commerciales d'Entandrophragma conduit à une méconnaissance des paramètres fondamentaux de la dynamique de leurs populations et, par conséquent, à la prise de décisions inadéquates par les gestionnaires forestiers (Doucet, 2003 ; FAO, 2011 ; Biwolé et al., 2012). De plus, les informations sur la distribution géographique, l'écologie, la taxonomie, la génétique et l'évolution des espèces d'Entandrophragma restent encore fragmentaires.

L'objectif général de cette étude est de faire une synthèse des connaissances acquises sur la taxonomie et l'écologie de toutes les espèces d'Entandrophragma, avec une attention particulière sur les principales espèces exploitées dans les forêts denses humides.

Les objectifs spécifiques sont les suivants :

- décrire l'évolution de leur taxonomie et leurs caractéristiques écologiques ;
- discuter des aspects pour lesquels les connaissances restent insuffisantes ou imprécises afin de suggérer des pistes de recherches futures en appui à l'amélioration de leur gestion durable.

\section{TAXONOMIE DU GENRE}

\subsection{Historique, synonymie et subdivision taxonomique}

Historique. Le nom de genre Entandrophragma a été proposé en 1894 par Casimir de Candolle dans sa "Monographie des Méliacées ». Son étymologie fait allusion à la particularité florale unique de ce genre dans la famille des Meliaceae : l'existence d'un disque sous l'ovaire et de dix véritables cloisons «phragma » qui forment des godets à la base du tube staminal (Tailfer, 1989). Des rapprochements ont été faits avec le genre américain Swietenia Jacq. en 1858 par Welwitsch, puis le genre asiatique Chukrasia A.Juss. par de Candolle en 1894. Ce dernier genre se distingue des Entandrophragma au niveau des fleurs par un tube staminal long et étroit avec l'absence de cloison interne et, surtout, par le fruit qui est une capsule dressée multisperme (Louis \& Fouarge, 1947).

Synonymie. Le genre Entandrophragma est aussi caractérisé par une importante évolution de la synonymie des noms des espèces décrites par différents auteurs, dont le recensement aboutit à un total de 36 à 37 espèces (Tableau 1). Cette synonymie taxonomique abondante pourrait trouver sa source, selon Louis \& Fouarge (1947), dans l'intérêt économique important du genre. Cet intérêt aurait conduit à un regain d'attention de la part des systématiciens qui, suite à la variabilité phénotypique au sein des espèces, auraient favorisé la 
Tableau 1. Synonymie et subdivision de 10 espèces d'Entandrophragma reconnues dans la base de données des plantes d'Afrique (Conservatoire et Jardin Botaniques de la Ville de Genève). Deux espèces supplémentaires sont répertoriées dans la base de données (CThe Plant List 2013 - Synonymy and subdivision of 10 Entandrophragma species recognized in the African Plant Database. Two additional species are mentionned in CThe Plant List 2013.

\begin{tabular}{|c|c|c|}
\hline $\begin{array}{l}\text { Section } \\
\text { (Louis \& Fouarge, 1947) }\end{array}$ & $\begin{array}{l}\text { Noms spécifiques actuellement acceptés } \\
\text { (www.ville-ge.ch/musinfo/bd/cjb/africa/, } \\
\text { 07/03/2015) }\end{array}$ & $\begin{array}{l}\text { Synonymes selon Louis \& Fouarge } \\
\text { (1947) et (www.ville-ge.ch/musinfo/bd/ } \\
\text { cjb/africa/,07/03/2015) }\end{array}$ \\
\hline Neoentandrophragma Harms & $\begin{array}{l}\text { E. utile (Dawe et Sprague) Sprague } \\
\text { (NC : sipo) }\end{array}$ & $\begin{array}{l}\text { E. roburoides Vermoesen (1921) } \\
\text { E. thomasii Ledoux (1932) } \\
\text { E. macrocarpum A.Chev. (1909) } \\
\text { Pseudocedrela utilis Dawe \& Sprague } \\
\text { (1906) }\end{array}$ \\
\hline Pseudoentandrophragma Harms & $\begin{array}{l}\text { E. cylindricum (Sprague) Sprague } \\
\text { (NC : sapelli) }\end{array}$ & $\begin{array}{l}\text { E. lebrunii Staner (1930) } \\
\text { E. angolense Auct. Non C.DC. } \\
\text { E.tomentosum A.Chev. ex Hutch. \& } \\
\text { Dalziel (1928) } \\
\text { E. rufum A.Chev. (1909) }\end{array}$ \\
\hline \multirow[t]{2}{*}{ Choriandra Harms } & E. palustre Staner (NC : -) & $\begin{array}{l}\text { Heimodendon tisserantii Sillans } \\
\text { Heimodendron tisserantii Sillans (1953) }\end{array}$ \\
\hline & E. candollei Harms (NC : kossipo) & $\begin{array}{l}\text { E. choriandrum Harms } \\
\text { E.ferruginea A.Chev. }\end{array}$ \\
\hline \multirow[t]{3}{*}{ Euentandrophragma Harms } & $\begin{array}{l}\text { E. angolense (Welw.) C.DC. } \\
\text { (NC : tiama blanc) }\end{array}$ & $\begin{array}{l}\text { E. casimiriana De Wild. \& Th. Dur. } \\
\text { E. candolleanum De Wild. \& Th. Dur. } \\
\text { (1899) } \\
\text { E. gregoireianum Staner (1930) } \\
\text { E. congoense (De Wild.) A.Chev. (*) } \\
\text { E. platanoides Vermoensen (1921) } \\
\text { E. leplaei Vermoense (1921) } \\
\text { E. congoensis (Pierre ex De Wild.) } \\
\text { A.Chev. } \\
\text { E. macrophyllum A.Chev. (1909) } \\
\text { E. pierrei A.Chev. (1909) (*) } \\
\text { E. cylindricum Auct. Non (Sprague) } \\
\text { Sprague } \\
\text { E. rederi Harms (1910) } \\
\text { E. septentrionale A.Chev. (1909) } \\
\text { Swietenia angolensis Welw. (1859) } \\
\text { Leioptux congensis Pierre ex De Wild. }\end{array}$ \\
\hline & $\begin{array}{l}\text { E. excelsum (Dawe et Sprague) Sprague } \\
\text { (NC : acajou de montagne) }\end{array}$ & $\begin{array}{l}\text { Pseudocedrela excelsa Dawe \& Sprague } \\
\text { E. speciosum Harms } \\
\text { E. gillardini Ledoux } \\
\text { E. stolzii Harms (1917) } \\
\text { E. deiningeri Harms (1917) }\end{array}$ \\
\hline & $\begin{array}{l}\text { E. delevoyi De Wild } \\
(\mathrm{NC}:-)\end{array}$ & E. lucens Hoyle (1932) \\
\hline \multirow[t]{3}{*}{ Wulfhorstia (C.DC.) Sprague } & $\begin{array}{l}\text { E. spicatum (C.DC.) Sprague } \\
\text { (NC : mutaco) }\end{array}$ & $\begin{array}{l}\text { Wulfhorstia spicata } \text { C.DC. (1900) } \\
\text { E. ekebergioides (Harms) Sprague } \\
\text { Wulfhorstia ekebergioides Harms (1903) }\end{array}$ \\
\hline & E. caudatum Sprague (NC : -) & Pseudocedrela caudata Sprague (1908) \\
\hline & E. bussei Harms (NC: -) & - \\
\hline
\end{tabular}

- : pas d'information - no information; NC : nom commercial - commercial name ; (*) : espèces acceptées par CThe Plant List 2013 - species accepted by CThe Plant List 2013, http://www.theplantlist.org/tpl1.1/record/kew-2789562, (20/12/2016), http://www. theplantlist.org/tpl1.1/record/kew-2789547, (20/12/2016). 
création parfois abusive d'espèces. En effet, une partie de ces espèces a été décrite et nommée par des auteurs différents ayant une connaissance insuffisante de la littérature scientifique de l'époque. L'amélioration progressive des connaissances scientifiques a permis de regrouper certaines de ces espèces en une seule et de supprimer ainsi ces synonymies, généralement anciennes (Tableau 1). Ainsi, le Conservatoire et Jardin Botaniques de la Ville de Genève (CJBG, 2012) reconnaissent actuellement seulement 10 espèces (Entandrophragma congoense [Pierre ex De Wild.] A.Chev. non incluse) dans toute l'aire africaine (Tableau 1). Parmi les espèces désormais reconnues, Entandrophragma angolense (Welw.) C.DC. dispose d'une synonymie très abondante, due probablement au polymorphisme relatif qu'elle manifeste, comme beaucoup de grands arbres à large aire de répartition. Une relative confusion persiste encore à ce jour concernant la synonymie de E. angolense et E. congoense, soutenue par Louis \& Fouarge (1947) et plus récemment par Chevallier et al. (2010). Par contre, Wilks \& Issembé (2000) ainsi que de Wilde (2015) reconnaissent que $E$. angolense et $E$. congoense sont des espèces distinctes. Cette distinction est aussi faite par les prospecteurs forestiers au Gabon et au Cameroun en les appelant respectivement «tiama blanc» (E. angolense : tronc gris-blanc à gris brun et tranche rose avec des raies blanchâtres) et «tiama noir » (E. congoense : tronc brun-noirâtre et tranche rose-rouge assez foncée ; Meunier et al., 2015). Pour la suite de cette revue, nous reconnaitrons $E$. congoense et E. angolense comme deux espèces à part entière, conformément à la Flore du Gabon (de Wilde, 2015). Ainsi, nous considérons qu'il y a 11 espèces d'Entandrophragma et non 10. Par ailleurs, des études utilisant des approches de génétique moléculaire plus poussées et couplées à d'autres critères botaniques, notamment la symétrie de la foliole et le nombre de nervures secondaires, seraient nécessaires pour lever certaines incertitudes en termes de différence entre les deux tiamas.

Subdivision. À l'exception de E.congoense pour laquelle des doutes subsistent, les espèces d'Entandrophragma ont été groupées en cinq sections (Tableau 1) par Louis \& Fouarge (1947). Cette subdivision est basée sur les caractères fertiles dont l'existence du disque sur l'ovaire, la nature des dix cloisons délimitant les dix godets à la base du tube staminal et la forme de la capsule. L'utilité d'une telle subdivision réside dans les liens interspécifiques qu'elle fait ressortir. Cependant, l'étude d'autres caractères, notamment écologiques, anatomiques et génétiques permettrait une meilleure description des liens/différences entre les espèces d'Entandrophragma.

\subsection{Clé d'identification botanique des espèces d'Entandrophragma}

Différentes clés d'identification botanique des espèces d'Entandrophragma ont été proposées (e.g. Staner, 1943 ; Wilks \& Issembé, 2000 ; de Wilde, 2015). Ces clés reposent respectivement sur la description de caractères végétatifs et reproducteurs (fleurs et fruits). Cependant, aucune flore ou guide de terrain ne propose une clé d'identification intégrant l'ensemble des espèces d'Entandrophragma dont Entandrophragma spicatum (C.DC.) Sprague, Entandrophragma caudatum (Sprague) Sprague et Entandrophragma bussei Harms, pourtant partiellement décrites. La présente synthèse bibliographique propose une seule clé basée sur la combinaison de ces deux principaux caractères (Encadré 1). L'identification des grands Entandrophragma fertiles semble assez aisée, mais on n'a pas toujours accès aux fleurs. Ainsi, le tableau 2 présente les critères végétatifs autorisant une identification des Entandrophragma de forêts denses humides, y compris pour les jeunes arbres non encore fructifères (e.g. Wilks \& Issembé, 2000 ; Meunier et al., 2015).

\section{DISTRIBUTION GÉOGRAPHIQUE}

Le genre Entandrophragma est exclusivement africain. Les limites des entités géographiques climatiquement homogènes généralement utilisées (région, sous-région, domaine, etc.) par chaque auteur (e.g. Louis \& Fouarge, 1947 ; White, 1986) dépendent du degré (dimension spatiale) et de la nature (dimension temporelle) des distributions observées sur la base des données floristiques et écologiques disponibles. Louis \& Fouarge (1947) utilisent le terme «domaine » (e.g. zambézien, oriental, etc.) pour localiser l'aire de distribution des Entandrophragma, tandis que White (1986) préfère le terme de «région » et Sépulchre et al. (2008), « subregion ». La présente revue préfère les concepts de White (1986) puisqu'ils sont les plus couramment utilisés et sont issus du processus classique d'endémisme de conservation des espèces qui a débouché sur la production de la carte des principales phytocories de l'Afrique et de Madagascar.

Le genre Entandrophragma présente deux principales distributions géographiques (Figure 1).

Le premier groupe est composé de six espèces à très larges distributions guinéo-congolaises : E.angolense (tiama blanc), E.congoense (tiama noir), Entandrophragma candollei Harms (kosipo), Entandrophragma cylindricum (Sprague) Sprague (sapelli), Entandrophragma palustre Staner et Entandrophragma utile (Dawe \& Sprague) Sprague (sipo). Sans inclure E.palustre qui se cantonne aux forêts marécageuses de la République Démocratique du 
1. Bourgeon terminal glabre ou glabrescent ; pétales glabres sur les 2 faces ; anthères exsertes, elliptiques, arrondies au sommet :

2. Folioles glabres, sauf la nervure médiane à la face inférieure ; pétiolules de 1-6 mm ; tube staminal à bords entiers, non épaissis E. angolense

3. Nervure médiane en dessous glabre sur les flancs ; réseau des nervilles saillant ; parfois à domaties à l'aisselle des nervures ; tube staminal 2-3 mm de longueur E. congoense

1. Bourgeon terminal tomenteux ou pubérulent ; pétales au moins velus ou pubérulents sur la face externe ; anthères exsertes ou incluses, elliptiques ou lancéolées :

4. Essence des forêts de montagne de l'Est E. excelsum

4. Essence des forêts hygrophiles :

5. Folioles glabres ou rarement à fine pubérulence sur les nervures à la face inférieure sans domaties cryptiques pubescentes; pétales réfratés à l'anthèse velus sur les deux faces :

6. Pétiolules de 1-5 mm de long, pubescents ; folioles au nombre de 10-18, à 15-22 paires nervures latérales ; pétales oblancéolés-oblongs, rétrécis à la base ; anthères exsertes, elliptiques, arrondies au sommet

E. candollei

6. Pétiolules de 7-10 mm de long, glabres ; folioles au nombre de 6-10, à 10-13 paires nervures latérales ; pétales ovales-oblongs, non rétrécis à la base ; anthères incluses, triangulaires et aigües au sommet ; essence de forêts marécageuses

5. Folioles à domaties cryptiques pubérulentes à l'aisselle des nervures latérales à la face inférieure :

7. Folioles à 10-16 paires latérales très proéminentes à la face inférieure, non anastomosées en arc vers le bord; ovaire à 6-8 ovules en deux séries E. palustre

7. Folioles à 6-9 paires de nervures latérales peu proéminentes à la face inférieure, anastomosées en arc vers le bord; anthères exsertes triangulaires terminées au sommet par une petite pointe ........E. cylindricum

8. Essence endémique de la Tanzanie, présente dans les fourrés décidus, la forêt claire et la savane arbustive :

9. Folioles ovales, asymétriques, légèrement pubescentes au-dessous ..................................................... bussei

8. Essence des forêts claires ou sempervirentes sèches :

10. Folioles glabres ; pétiolules de 15-35 mm ; tube staminal à bords lobulés, épaissis au sommet

11. Folioles en 3-7 paires, jusqu'à 110 x 70 mm, largement oblongues ou obovales-oblongues ; surface inférieure densément pubérulente, nervure formant un réseau apparent E. delevoyi

12. Fruit : une capsule ligneuse, atteignant $15 \mathrm{~cm}$ de long, se divisant en 5 valves, qui se courbent en arrière montrant les graines ailées attachées à une colonne centrale E. spicatum 13. Fruits à déhiscence basilaire :

14. Valves très minces, 1-1,5 mm d'épaisseur, columelle jaune-paille marbrée de taches brun foncé E. caudatum

14. Valves de 4-5 mm ; columelle non marbrée :

15. Columelle brune acajou ; graines carrées de $1,5 \mathrm{~cm}$ de côté E. delevoyi

15. Columelle brune-chocolat; graines ovoïdes de $2,5 \mathrm{~cm}$ de long et $1,5 \mathrm{~cm}$ de large E. angolense 13. Fruits à déhiscence apicale :

16. Fruits claviformes ou ellipsoïdes, 18-28 cm de long, 5-7 cm de large ; columelle non stipitée E. excelsum

16. Fruits non claviformes, ne dépassant pas $5 \mathrm{~cm}$ de large; columelle à stipe grêle ou $5 \mathrm{~cm}$ non stipitée ne portant pas à la base les vestiges des valves :

17. Fruits non stipités à la base, $7-15 \mathrm{~cm}$ de long et $2,5-3 \mathrm{~cm}$ de large ; columelle cylindrique à bords ailés sur $1 \frac{1}{2}$ longueur E. utile

17. Fruits stipités à la base :

18. Fruits de $17-23 \mathrm{~cm}$ de long; columelle à stipe de $3-5 \mathrm{~cm}$ de long E.cylindricum 18. Fruits de 22-27 cm de long; columelle à stipe de $8-10 \mathrm{~mm}$ de long E. candollei E. palustre

Encadré 1. Clé simple et globale d'identification botanique d'espèces d'Entandrophragma basée sur les caractères végétatifs et reproducteurs (fleurs et fruits) (adapté d'après Staner, 1943 ; Roux, 2003 ; Lemmens, 2008 ; de Wilde, 2015 ; Burrows et al., 2017) - Global simple botanical identification key of the Entandrophragma species based on the vegetative and reproductive traits (flowers and fruits) (Staner, 1943; Roux, 2003; Lemmens, 2008; de Wilde, 2015; Burrows et al., 2017).

Congo et de la République du Congo et probablement d'autres régions d'Afrique centrale (Lemmens, 2008), les autres espèces croissent dans les forêts denses humides sempervirentes et semi-décidues (Meunier et al., 2015) où E. cylindricum et E. angolense sont plus abondantes localement qu'E. candolei et E. utile (Doumenge et al., 2010). Ces espèces s'étendent en une large bande au nord et au sud de l'équateur à partir du littoral atlantique jusqu'au versant occidental de la dorsale du Kivu à l'est, en passant par le bassin du Congo (White, 1986). Celle présentant une distribution la plus étendue parmi ces quatre espèces est E. angolense (Figure 1). Elle est certainement confondue avec son synonyme (E. congoense) par le CJBG. 
Tableau 2. Différenciation botanique des principales espèces d'Entandrophragma des forêts denses humides en Afrique (adapté de Wilks \& Issembé, 2000 ; Meunier et al., 2015) - Botanical differentiation of main Entandrophragma rain forest species in Africa (adapted from Wilks \& Issembé, 2000; Meunier et al., 2015).

\begin{tabular}{|c|c|c|c|c|c|}
\hline $\begin{array}{l}\text { Caractère } \\
\text { distinctif }\end{array}$ & E. angolense & E. congoense & E. candollei & E. cylindricum & E. utile \\
\hline Arbre & \multicolumn{2}{|c|}{$\begin{array}{l}\text { atteignant } 50 \mathrm{~m} \text { de haut et } 1,5 \mathrm{~m} \text { de } \\
\text { diamètre, avec des contreforts ou des } \\
\text { empattements à la base cylindrique ou à } \\
\text { empattements }\end{array}$} & $\begin{array}{l}\text { atteignant } 60 \mathrm{~m} \\
\text { de haut et } 3 \mathrm{~m} \\
\text { de diamètre, à } \\
\text { cime puissante } \\
\text { charpentée par de } \\
\text { grosses branches } \\
\text { sinueuses, muni de } \\
\text { contreforts arrondis } \\
\text { ou d'empattements } \\
\text { qui s'étendent } \\
\text { souvent loin de } \\
\text { l'arbre }\end{array}$ & $\begin{array}{l}\text { atteignant } 60 \mathrm{~m} \\
\text { de haut et plus de } \\
2 \mathrm{~m} \text { de diamètre, } \\
\text { à cime arrondie. Il } \\
\text { présente à la base } \\
\text { des empattements } \\
\text { ou des contreforts } \\
\text { parfois très } \\
\text { développés }\end{array}$ & $\begin{array}{l}\text { pouvant dépasser } \\
60 \text { m de haut et } \\
2 \text { m de diamètre, } \\
\text { à puissante cime } \\
\text { hémisphérique. } \\
\text { Il est muni } \\
\text { d'empattements ou } \\
\text { de contreforts à la } \\
\text { base }\end{array}$ \\
\hline Tronc & $\begin{array}{l}\text { droit et cylindrique } \\
\text { lisse à écailleux, } \\
\text { est souvent marqué } \\
\text { de dépressions } \\
\text { irrégulières et de } \\
\text { lenticelles }\end{array}$ & $\begin{array}{l}\text { plus foncé } \\
\text { aux fissures } \\
\text { verticales et } \\
\text { écailles allongées, } \\
\text { normalement } \\
\text { sans contreforts, } \\
\text { une base plus } \\
\text { cylindrique }\end{array}$ & $\begin{array}{l}\text { droit, lisse chez } \\
\text { les jeunes arbres, } \\
\text { et marqué de } \\
\text { nombreuses } \\
\text { dépressions } \\
\text { profondes donnant } \\
\text { un aspect piqué, } \\
\text { avec des écailles, } \\
\text { des crêtes sinueuses } \\
\text { et des cicatrices }\end{array}$ & $\begin{array}{l}\text { droit, gris à brun, } \\
\text { est presque lisse } \\
\text { chez les jeunes } \\
\text { arbres. Il est } \\
\text { ensuite couvert } \\
\text { d'écailles, de } \\
\text { lenticelles et } \\
\text { de nombreuses } \\
\text { dépressions chez } \\
\text { les très vieux } \\
\text { arbres, lui donnant } \\
\text { un aspect piqué }\end{array}$ & $\begin{array}{l}\text { droit, gris argenté } \\
\text { à gris-jaune, } \\
\text { marqué de } \\
\text { fissures verticales, } \\
\text { d'écailles } \\
\text { allongées et de } \\
\text { lenticelles }\end{array}$ \\
\hline Tranche & $\begin{array}{l}\text { rose avec des raies } \\
\text { blanchâtres, elle } \\
\text { dégage une très } \\
\text { légère odeur de } \\
\text { parfum }\end{array}$ & $\begin{array}{l}\text { rose-rouge, } \\
\text { finement fibreuse, } \\
\text { virant au brun, } \\
\text { cassante, } \\
\text { épaisseur de } 10- \\
30 \mathrm{~mm} \text {, couche } \\
\text { interne blanchâtre } \\
\text { à orangée }\end{array}$ & $\begin{array}{l}\text { rose-rouge à } \\
\text { blanchâtre avec } \\
\text { des granules jaune } \\
\text { orangé. On retrouve } \\
\text { parfois des amas } \\
\text { de gomme sur de } \\
\text { vieilles blessures }\end{array}$ & $\begin{array}{l}\text { brun rosâtre et vire } \\
\text { très rapidement au } \\
\text { brun, elle dégage } \\
\text { une très forte odeur } \\
\text { parfumée }\end{array}$ & $\begin{array}{l}\text { rose-rouge, } \\
\text { parfois avec des } \\
\text { raies blanches, } \\
\text { est peu odorante. } \\
\text { On y remarque } \\
\text { une pellicule } \\
\text { blanchâtre entre } \\
\text { l'écorce morte et } \\
\text { l'écorce vivante, et } \\
\text { sa couche interne } \\
\text { blanchâtre vire } \\
\text { lentement au brun }\end{array}$ \\
\hline Feuilles & $\begin{array}{l}\text { regroupées à } \\
\text { l'extrémité des } \\
\text { branches, sont } \\
\text { composées et } \\
\text { munies de } 4 \text { à } 9 \\
\text { paires de folioles } \\
\text { larges opposées } \\
\text { ou presque, dont } \\
\text { l'acumen a des } \\
\text { bords repliés }\end{array}$ & $\begin{array}{l}\text { acumen souvent } \\
\text { « en cuillère » } \\
\text { (bord replié en } \\
\text { forme de cuillère), } \\
\text { 8-18 paires de } \\
\text { nervures latérales, } \\
\text { forme plus } \\
\text { allongée que } \\
\text { le tiama blanc, } \\
\text { longueur } \\
7-15 \mathrm{~cm}, \text { rachis } \\
\text { à } 8 \text {-10 paires de } \\
\text { folioles opposées } \\
\text { ou presque }\end{array}$ & $\begin{array}{l}\text { regroupées à } \\
\text { l'extrémité des } \\
\text { branches, sont } \\
\text { composées et } \\
\text { munies de } 5 \text { à } 9 \\
\text { paires de folioles } \\
\text { à surface ondulée, } \\
\text { et parcourue par } \\
\text { de nombreuses } \\
\text { nervures latérales } \\
\text { (entre } 15 \text { et } 25 \\
\text { paires) }\end{array}$ & $\begin{array}{l}\text { regroupées } \\
\text { à l'extrémité } \\
\text { des branches, } \\
\text { composées et } \\
\text { munies de } 6 \text { à } 10 \\
\text { paires de folioles, } \\
\text { opposées ou } \\
\text { alternes. Elles sont } \\
\text { dissymétriques, } \\
\text { marquées par un } \\
\text { réseau de nervilles } \\
\text { saillantes sur les } \\
\text { deux faces, avec } \\
\text { la face supérieure } \\
\text { luisante à l'état sec, } \\
\text { et parfois avec des } \\
\text { touffes de poils à la } \\
\text { base des nervures }\end{array}$ & $\begin{array}{l}\text { composées, munies } \\
\text { de } 6 \text { à } 12 \text { paires de } \\
\text { folioles allongées, } \\
\text { dissymétriques, } \\
\text { marquées de } \\
\text { touffes de poils } \\
\text { à l'aisselle des } \\
\text { nervures latérales, } \\
\text { ces dernières étant } \\
\text { également plus ou } \\
\text { moins poilues }\end{array}$ \\
\hline
\end{tabular}


Tableau 2 (suite). Différenciation botanique des principales espèces d'Entandrophragma des forêts denses humides en Afrique (adapté de Wilks \& Issembé, 2000 ; Meunier et al., 2015) - Botanical differentiation of main Entandrophragma rain forest species in Africa (adapted from Wilks \& Issembé, 2000; Meunier et al., 2015).

\begin{tabular}{|c|c|c|c|c|c|}
\hline $\begin{array}{l}\text { Caractère } \\
\text { distinctif }\end{array}$ & E. angolense & E. congoense & E. candollei & E. cylindricum & E. utile \\
\hline Fleurs & verdâtres et petites & pas d'information & vert-jaune & $\begin{array}{l}\text { vert pâle et de } \\
\text { petite dimension }\end{array}$ & vert jaunâtre \\
\hline Fruits & $\begin{array}{l}\text { grosses capsules } \\
\text { allongées qui } \\
\text { rappellent de gros } \\
\text { cigares (jusqu'à } \\
22 \text { x } 5 \mathrm{~cm} \text { ), à } 5 \\
\text { valves ligneuses } \\
\text { couvertes de } \\
\text { lenticelles, qui } \\
\text { s'ouvrent par la } \\
\text { base et restent } \\
\text { fixées au sommet }\end{array}$ & $\begin{array}{l}\text { des fruits noirâtres } \\
\text { s'ouvrant } \\
\text { tardivement, on } \\
\text { les trouve souvent } \\
\text { entiers au sol }\end{array}$ & $\begin{array}{l}\text { capsules allongées } \\
\text { brunes qui } \\
\text { rappellent de gros } \\
\text { cigares (jusqu'à } \\
23 \text { x } 5 \mathrm{~cm} \text { ) parfois } \\
\text { lenticellées, à } 5 \\
\text { valves ligneuses } \\
\text { qui s'ouvrent par le } \\
\text { sommet }\end{array}$ & $\begin{array}{l}\text { noirs au sol, sont } \\
\text { des capsules } \\
\text { allongées qui ont } \\
\text { l'aspect de gros } \\
\text { cigares (jusque } \\
22 \times 4 \mathrm{~cm} \text { ), à } 5 \\
\text { valves s'ouvrant } \\
\text { par le sommet } \\
\text { et une surface } \\
\text { relativement lisse }\end{array}$ & $\begin{array}{l}\text { grosses capsules } \\
\text { (jusque } 28 \text { x } \\
7 \mathrm{~cm} \text { ), à valves } \\
\text { très épaisses, à } \\
\text { surface brun-noir } \\
\text { très verruqueuse } \\
\text { s'écartant par le } \\
\text { sommet et restant } \\
\text { fixées à la base }\end{array}$ \\
\hline Graines & aplaties et ailées & ailées & aplaties et ailées & & $\begin{array}{l}\text { plates munies } \\
\text { d'une longue aile }\end{array}$ \\
\hline
\end{tabular}

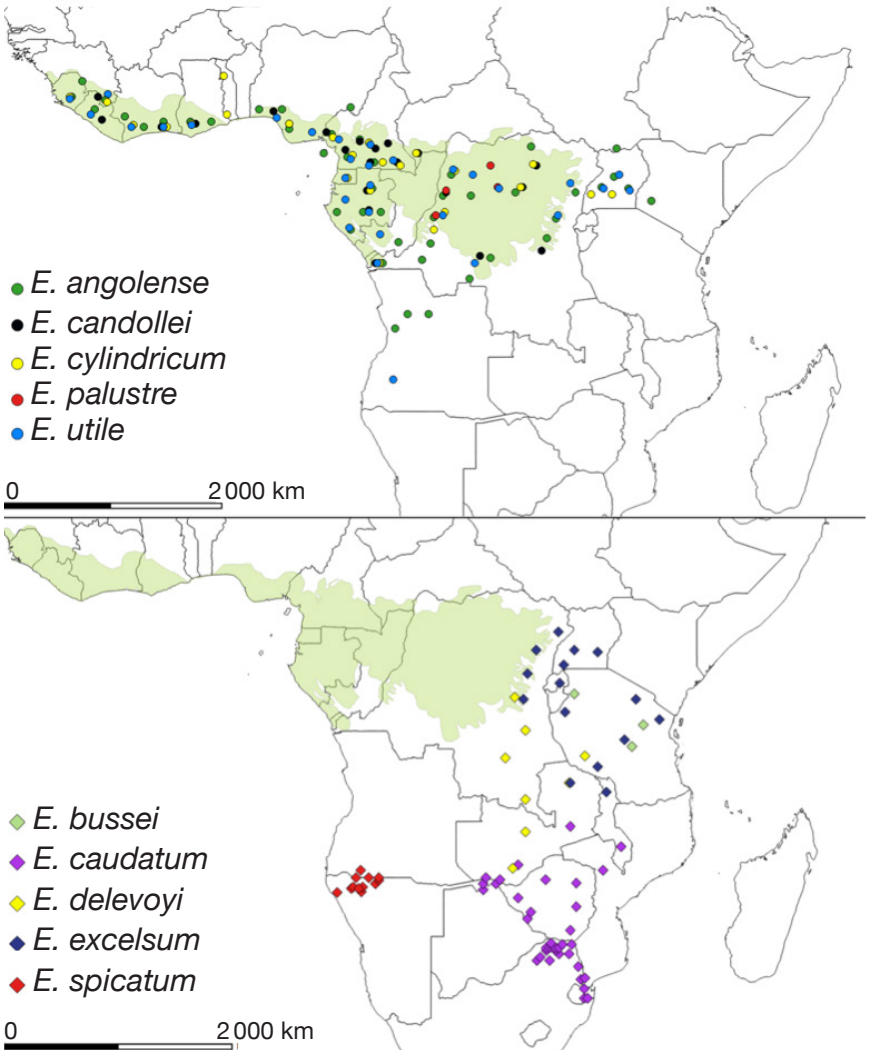

Figure 1. Répartition géographique des espèces du genre Entandrophragma (carte adaptée d'après la base de données des Conservatoire et Jardin Botaniques de la Ville de Genève), qui assimile E. congoense à E. angolense - Natural range of the species of genus Entandrophragma (map adapted from the database of the Conservatoire et Jardin botaniques de la Ville de Genève), which considers E. congoense as a synonym of $\mathrm{E}$. angolense.
Le second groupe comprend cinq espèces à distributions zambézienne et afromontagnarde (Entandrophragma bussei, Entandrophragma caudatum, Entandrophragma delevoyi De Wild, Entandrophragma spicatum et Entandrophragma excelsum) (Dawe \& Sprague) Sprague (Louis \& Fouarge, 1947 ; White, 1986; Malaise, 1993 ; Lemmens, 2008). Entandrophragma excelsum est une espèce à distribution à la fois «zambézienne » et «afromontagnarde » où elle se limite aux forêts de montagne (Louis \& Fouarge, 1947) et est présente dans l'est de la RDC, au Rwanda, au Burundi, en Ouganda, en Tanzanie, au Malawi et en Zambie (Lemmens, 2008), tandis qu' $E$. caudatum, $E$. delevoyi et $E$. spicatum, dites «zambéziennes »se retrouvent dans les forêts claires ou sempervirentes sèches (Louis \& Fouarge, 1947 ; Lemmens, 2008). Il est précisé que seule E. delevoyi (à distribution zambézienne et afromontagnarde) est principalement caractéristique des forêts denses sèches (Dikumbwa, 1990 ; Malaise, 1993 ; Malaise et al., 2000). La figure 1 montre que sa distribution est plus large en RDC et en Zambie, et restreinte en Tanzanie. Par contre, E. caudatum est répartie au sud de la Zambie et du Malawi, au nord-est de l'Afrique du Sud, au Zimbabwe, Mozambique et Botswana (Burrows et al., 2017), alors qu'E. spicatum est confinée au sud de l'Angola et au nord de la Namibie (Figure 1). Par ailleurs, E. bussei (à distribution afromontagnarde) est endémique de la Tanzanie où elle est présente dans les fourrés décidus, la forêt claire et la savane arbustive (Lemmens, 2008). 


\section{PHYLOGÉNIE ET GÉNÉTIQUE}

\subsection{Phylogénie}

Depuis plus d'une dizaine d'années, de nombreuses études moléculaires ont permis de clarifier les relations phylogénétiques au sein de la famille des Meliaceae (Muellner et al., 2006; Muellner et al., 2009; Pennington et al., 2010 ; Muellner et al., 2011). Des révisions taxonomiques notamment pour les genres Carapa Aubl., Cedrela et Leplaea (ex-Guarea) ont été réalisées (Kenfack, 2011 ; Muellner et al., 2011). Selon une étude phylogénétique récente sur les Meliaceae, le genre Entandrophragma est considéré proche du genre Cedrela dont il aurait divergé lors de l'Oligocène il y a près de 30 millions d'années (Koenen et al., 2015). Malgré ces études, les relations phylogénétiques et l'origine de la diversification des espèces du genre Entandrophragma restent encore inconnues.

\subsection{Nombres chromosomiques et marqueurs génétiques}

Le niveau de ploïdie est assez homogène au sein du genre Entandrophragma $(2 \mathrm{n}=36)$, bien que Mangenot \& Mangenot (1962) rapportent une duplication du niveau de ploïdie chez E. angolense, E. cylindricum et $E$. utile $(2 \mathrm{n}=36$ ou 72$)$. L'existence de cytotypes polyplö̈des n'a toutefois pas été confirmée chez E. cylindricum par l'utilisation de marqueurs microsatellites depuis leur développement (Garcia et al., 2004). Ces marqueurs génétiques ont ouvert la voie à différentes études sur le système de reproduction et la dispersion des gènes chez E.cylindricum (Lourmas et al., 2007 ; Monthe et al., 2017a), ainsi que sur l'identification des pieds exploités en vue de contrôler leur commerce (Jolivet \& Degen, 2012). Ils ont notamment permis de démontrer qu'E. cylindricum est principalement allogame et que le pollen, tout comme les graines, sont en moyenne dispersés à plus de $600 \mathrm{~m}$ (Lourmas et al., 2007 ; Monthe et al., 2017a). Le récent développement de marqueurs microsatellites pour d'autres espèces d'Entandrophragma (e.g. E.candollei et E.utile) (Monthe et al., 2017b) permettra très probablement une amélioration des connaissances génétiques de ces taxons (e.g. analyse de l'impact de l'exploitation sur les populations sensu Lourmas et al., 2007).

\section{5. ÉCOLOGIE ET SYLVICULTURE}

\subsection{Niches climatiques, préférences édaphiques et habitat}

Niches climatiques. Aucune analyse globale n'a jamais été réalisée au sujet des niches climatiques des
Entandrophragma dans toute leur aire de répartition (Doumenge etal.,2010).En effet, à partird'informations issues de spécimens d'herbiers, d'inventaires forestiers et autres relevés de terrain, Doumenge et al. (2010) ont proposé une méthode permettant de modéliser les niches climatiques qu'ils ont appliquées aux quatre espèces d'Entandrophragma guinéo-congolaises les plus exploitées: E.angolense, E.candollei, E.cylindricum et E. utile. Hormis E.palustre qui préfère les zones les plus humides de la cuvette congolaise, cette approche montre que les espèces des forêts denses humides semblent davantage présentes sous des conditions d'humidité intermédiaire, évitant les climats les plus humides. Un second groupement est constitué des espèces d' "environnements plus secs » (E.bussei, E. caudatum, E. delevoyi et E. spicatum). Celles-ci s'acclimatent mieux dans des zones sèches et montrent une nette séparation d'E. excelsum, laquelle se caractérise par des préférences climatiques de haute altitude, généralement entre $1300 \mathrm{~m}$ et $2100 \mathrm{~m}$ (Louis \& Fouarge, 1947 ; Lemmens, 2008).

Des études sur la caractérisation des niches climatiques des espèces d'Entandrophragma demeurent insuffisantes et méritent d'être régulièrement approfondies pour tenir compte de l'évolution du climat récent et futur sur toute leur aire de répartition.

Préférences édaphiques et habitat. Les habitats des différentes espèces d'Entandrophragma se distingueraient par des propriétés physico-chimiques du sol. D'après Hall et al. (2004), dans les forêts du sud-ouest de la République Centrafricaine (RCA), la présence des cinq principales espèces d'Entandrophragma (E.angolense, E.congoense, E. candollei, E.cylindricum et E. utile) des forêts denses humides est corrélée à la composition chimique du sol. Par exemple, seule E. candollei tolère des sols très peu fertiles. Entandrophragma angolense et E. cylindricum sont caractéristiques des sols à $\mathrm{pH}$ atypiquement élevé alors qu'à l'opposé, E. candollei est limitée à des sols particulièrement acides. Ces analyses mériteraient d'être étendues à d'autres espèces du genre et à d'autres régions où plusieurs espèces cohabitent.

En outre, des associations mycorhiziennes à arbuscules et vésicules ont été enregistrées chez les principales espèces d'Entandrophragma exploitées par les compagnies forestières locales (Onguene \& Kuyper, 2001). L'inoculation mycorhizienne semble ainsi favoriser la mise en place des semis de E. utile (Mujuni, 2008).

\subsection{Tempérament et sylviculture}

Tempérament.La tolérance à l'ombre au stade juvénile des espèces d'Entandrophragma les plus exploitées en 
forêts denses humides (sapelli, sipo, kosipo et tiama blanc) a été diversement décrite selon les auteurs. Ces espèces sont ainsi qualifiées de sciaphiles (Louis \& Fouarge, 1947), tolérantes à l'ombre (Hall et al., 2003) ou de semi-héliophiles (Meunier et al., 2015 ; Doucet et al., 2016). Bien qu'il ressorte de ces travaux un consensus vers une certaine tolérance à l'ombrage (le tempérament semi-héliophile de ces espèces est actuellement admis), il existe néanmoins des nuances entre espèces (kosipo, sipo, sapelli et tiama blanc) établies par certains auteurs. Ces nuances de besoin en lumière peuvent aussi être vérifiées par une étude plus détaillée des traits ligneux en quantifiant les diamètres des vaisseaux de la moelle à l'écorce du bois des espèces respectives (Beeckman, 2016).

D'autre part, pour les espèces du genre autres que celles exploitées dans des forêts denses humides (E.bussei, E.caudatum, E. delevoyi, E. excelsum et E. spicatum), le tempérament est moins connu.

Sylviculture. Une connaissance insuffisante du tempérament de chacune des espèces d'Entandrophragma freine également la mise en œuvre de techniques sylvicoles, par exemple pour optimiser les reboisements. En effet, les expériences conduites par Hall (2008) en RCA et par Doucet et al. (2016) au Cameroun montrent que les techniques sylvicoles utilisées pour les régénérations des espèces d'Entandrophragma sont confrontées à des taux de mortalité élevés. Plusieurs techniques sylvicoles, notamment la méthode de régénération naturelle par coupe d'enrichissement et d'abri unique, la méthode des layons et celle d'enrichissement par semis direct, appliquées pour le renouvellement des Entandrophragma, n'ont pas donné des résultats très satisfaisants. D'autres techniques pour améliorer leur régénération ont été testées comme la création de petites trouées de taille intermédiaire dans les forêts exploitées et l'apport de semences afin d'obtenir une meilleure régénération (Hall et al., 2003 ; Makana \& Thomas, 2004). Néanmoins, la pertinence de ces méthodes de régénération est remise en question par de nouvelles études dont les résultats n'ont pas été satisfaisants avec ces espèces (Fayolle et al., 2015 ; Doucet et al., 2016). Tous les sapellis observés au Cameroun par Owona Ndongo et al. (2009) ont présenté de nombreuses difficultés dues à des piqûres d'insectes lors de la croissance juvénile, ce qui expliquerait notamment leur retard initial de croissance. Selon Eckebil et al. (2017), la sylviculture d'E. cylindricum n'en est qu'à son début. En effet, il y a encore lieu d'approfondir la recherche sylvicole sur toutes les espèces d'Entandrophragma dans leurs zones de répartition.

\subsection{Phénologie et diamètre de fructification}

Phénologie. D'une manière générale, les études sur la phénologie n'ont été menées que sur certaines espèces d'Entandrophragma (E.angolense, E.candollei, E. cylindricum, E. utile, E. delevoyi et E. caudatum) et plus rarement et de manière insuffisante sur d'autres (e.g. E. bussei, E. excelsum et E. spicatum).

En effet, Louis \& Fouarge (1947) précisent qu'en RDC, les Entandrophragma des forêts denses humides (E. angolense, E. candollei, E. cylindricum, E. palustre et $E$. utile) sont reconnues comme étant caducifoliées, à l'exception d'E. palustre qui est sempervirente. La période de défeuillaison correspond approximativement à la saison de fléchissement des précipitations (début de la grande saison sèche). Dans les forêts de l'Afrique de l'Ouest, quelques études phénologiques (Taylor, 1960 ; Voorhoeve, 1965 ; Poorter et al., 2004) décrivent les périodes de floraison et fructification des quatre principales espèces les plus exploitées (kosipo, sipo, sapelli et tiama blanc) (Tableau 3). La phénologie des Entandrophragma est a priori variable suivant les régions. En Afrique centrale, et spécifiquement en République du Congo (RC), Gillet et al. (2008) renseignent que le capital foliaire d'E. candollei se reconstitue de septembre à octobre après l'attaque des chenilles; les fleurs sont présentes en décembre et la fructification a lieu en mars-avril. Chez E. cylindricum (semi-décidu), le renouvellement foliaire s'effectue en aout (Eckebil et al., 2017) et en septembre après l'attaque de chenilles comestibles Imbrasia oyemensis (Gillet et al., 2008).

Tableau 3. Phénologie des espèces d'Entandrophragma en Afrique de l'ouest (principalement en Côte d'Ivoire, Libéria et Ghana) - Phenology of Entandrophragma species in West Africa (mainly in Côte d'Ivoire, Liberia and Ghana).

\begin{tabular}{llllll}
\hline Espèce & Nom commercial & Feuillage & Période de floraison & $\begin{array}{l}\text { Période de } \\
\text { fructification }\end{array}$ & Références \\
\hline E. angolense & Tiama blanc & $\begin{array}{l}\text { Décidu (septembre- } \\
\text { novembre) }\end{array}$ & Décembre & Juillet-septembre & $\begin{array}{l}\text { Taylor, 1960; } \\
\text { Voorhoeve, } 1965\end{array}$ \\
E. candollei & Kosipo & Décidu & Novembre-décembre & Mai-aout & Voorhoeve, 1965 \\
E. cylindricum & Sapelli & Décidu & Février-mars & Mai-aout & Taylor, $1960 ;$ \\
& & Décidu & Janvier-février & Décembre-mars & Voorhoeve, 1965 \\
\hline E. utile & Sipo & & & &
\end{tabular}


Chez E. delovoyi des forêts denses sèches de la RDC, le maximum de la chute des feuilles est observé au mois de septembre et la chute conjuguée des fruits et des graines est généralement observée au cours du mois de janvier (Dikumbwa, 1990). Il est par ailleurs précisé que la période de floraison chez E. caudatum va de septembre à octobre (Burrows et al., 2017).

Diamètre de fructification. Les données quantitatives concernant le diamètre minimal de fructification $(\mathrm{DMF})$ et le diamètre de fructification régulière (DFR) des espèces d'Entandrophragma des forêts denses humides (Tableau 4) demeurent encore fragmentaires. Le DMF est celui considéré comme le seuil à partir duquel on observe les premières floraisons et fructifications qui se traduisent néanmoins par une faible production de diaspores. Ce diamètre est aussi appelé diamètre minimum de fertilité. Le DFR est celui à partir duquel on assiste à une fructification efficace et régulière (Sépulchre et al., 2008). Ce dernier diamètre est surtout un outil d'aide à la détermination $\mathrm{du}$ diamètre minimal d'exploitabilité d'une essence (Menga et al., 2012) car il est indispensable que les arbres ne soient exploités (s'ils sont bien conformés) que s'ils dépassent largement ce diamètre afin d'assurer leur régénération et la pérennité de l'espèce. Si le DMF est déterminé pour une partie de ces espèces, le DFR demeure encore globalement méconnu (Durrieu de Madron \& Daumerie, 2004 ; Menga et al., 2012). Les diamètres minimums légaux d'exploitation (DME) sont un outil fondamental d'aménagement durable qui tient compte de la structure diamétrique des populations d'arbres et de la nécessité de préserver de l'abattage la fraction de semenciers devant assurer la régénération (Durrieu de Madron et al., 1998 ; Eckebil et al., 2017). Le DME est alors comparé au DMF et au DFR dès lors que l'on s'intéresse à la gestion raisonnée des populations d'une ou plusieurs espèces. De telles comparaisons devraient ainsi servir à réviser, si nécessaire, ces seuils d'exploitation.

Le tableau 4 nous montre que les DME des principalesessences commerciales d'Entandrophragma ont été établis sur des bases empiriques par les administrations forestières nationales car ils varient fortement d'un pays à l'autre : par exemple de 60 à $110 \mathrm{~cm}$ pour E. candolei entre la Côte d'Ivoire et le Ghana.

\subsection{Dissémination, régénération naturelle et structure des populations}

Dissémination. Les espèces d'Entandrophragma sont toutes anémochores (Louis \& Fouarge, 1947 ; Medjibe \& Hall, 2002 ; Poorter et al., 2004 ; Doucet et al., 2016). Les fruits capsulaires de ces espèces sont à déhiscence septifrage en cinq valves ligneuses. Ces fruits contiennent des graines pourvues d'une aile unique qui se dispersent en tournoyant sous l'effet du vent (Louis \& Fouarge, 1947). La quasi-totalité des graines issues des fruits sont de type «planeur lourd» et tombent généralement à proximité immédiate des semenciers (Medjibe \& Hall, 2002). Cependant, la dispersion des samares d'Entandrophragma devrait être vérifiée par des nouvelles mesures de dispersion (identification de plantules installées). En effet, une étude récente basée sur des méthodes moléculaires montre que la dispersion de ces samares par le vent est plus importante que ce que l'on pensait $( \pm 600 \mathrm{~m})$ (Monthe et al., 2017a). Cette étude montre aussi que les samares qui se trouvent proches du pied mère suite à une faible dispersion subiraient peut-être une plus forte prédation. Cette dernière est occasionnée par des insectes foreurs e.g. Hypsipyla (Poorter et al., 2004 ; de la Mensbruge, 1966), par des rongeurs et/ou ongulés (Poorter et al., 2004) attirés notamment par la forte teneur en huile des graines d'Entandrophragma (Louis \& Fouarge, 1947).

Régénération naturelle. La faiblesse du pouvoir germinatif des graines des Entandrophragma (de la Mensbruge, 1966), leur prédation (Louis \& Fouarge, 1947 ; de la Mensbruge, 1966), une fructification quelque peu imprévisible au cours d'une année (Hall, 2011), une floraison qui semble rythmique supraannuelle avec des années de meilleure fructification (Gillet et al., 2008) sont autant de raisons pouvant expliquer qu'il est rare de rencontrer une abondante régénération naturelle des Entandrophragma. Cette rareté de la régénération naturelle varie d'une espèce d'Entandrophragma à l'autre, mais elle est presque générale à toutes les espèces du genre (Lemmens, 2008).

Structure des populations. La courbe de distribution des effectifs par classe de diamètre est un indicateur de la structure de la population et du niveau de régénération. La structure des populations avec une courbe en exponentielle décroissante indique une bonne régénération, alors qu'une courbe en cloche témoigne une faible régénération. Entre ces deux extrêmes, il y a de nombreuses courbes intermédiaires dont l'interprétation est souvent délicate, notamment en raison de l'histoire de la forêt et de la précision de l'inventaire (Daïnou \& Doucet, 2010). Plusieurs études préparatoires à la rédaction de plans d'aménagements (FRM， 2007 ; FRM， 2009; Nature+, 2011) montrent que la structure des populations d'espèces d'Entandrophragma varie en fonction notamment du degré de la régénération naturelle, de la densité, des effectifs et des volumes des tiges inventoriées ainsi que des conditions écologiques de chaque espèce. La structure des populations de sapelli, par exemple, est 
Tableau 4. Diamètre minimum de fructification (DMF), diamètre de fructification régulière (DFR) et diamètre minimum d'exploitation (DME) des essences d'Entandrophragma - Average fruiting diameter, regular fruiting diameter, minimum logging diameter (DME) of Entandrophragma species.

\begin{tabular}{|c|c|c|c|c|c|c|}
\hline $\begin{array}{l}\text { Nom } \\
\text { commercial }\end{array}$ & $\begin{array}{l}\text { Nom } \\
\text { scientifique }\end{array}$ & $\begin{array}{l}\text { DMF } \\
(\mathrm{cm})\end{array}$ & $\begin{array}{l}\text { DFR } \\
(\mathrm{cm})\end{array}$ & $\begin{array}{l}\text { DME } \\
(\mathrm{cm})\end{array}$ & Pays & Référence \\
\hline \multirow[t]{9}{*}{ Kosipo } & E. candollei & 40 & & 80 & $\mathrm{RCA}$ & Yalibanda, 1999 \\
\hline & & & 85 & 70 & Ouganda & Plumptre, 1995 \\
\hline & & & & 110 & Ghana & Nyunaï, 2008 \\
\hline & & & & 90 & Libéria & \\
\hline & & & & 60 & $\mathrm{CI}$ & \\
\hline & & & & 80 & Gabon & \\
\hline & & & & 80 & $\mathrm{RC}$ & \\
\hline & & & & 80 & Cameroun & \\
\hline & & & & 80 & $\mathrm{RDC}$ & \\
\hline \multirow[t]{11}{*}{ Sapelli } & E. cylindricum & & 75 & 80 & RCA & Durrieu de Madron \& Daumeurie, 2004 \\
\hline & & & 85 & & Ouganda & Plumptre, 1995 \\
\hline & & 35 & & 80 & $\mathrm{RCA}$ & Petrucci et al., 1995 \\
\hline & & 55 & & 80 & $\mathrm{RCA}$ & Yalibanda, 1999 \\
\hline & & 40 & 55 & 90 & Gabon & Sépulchre et al., 2008 \\
\hline & & & & 60 & $\mathrm{CI}$ & Palla et al., 2002 \\
\hline & & & & 110 & Ghana & \\
\hline & & & & 90 & Libéria & \\
\hline & & & & 80 & $\mathrm{RC}$ & \\
\hline & & & & 100 & Cameroun & \\
\hline & & & & 80 & $\mathrm{RDC}$ & \\
\hline \multirow[t]{8}{*}{ Sipo } & E. utile & & 85 & 90 & Gabon & Sépulchre et al., 2008 \\
\hline & & & & 110 & Ghana & Mujuni, 2008 \\
\hline & & & & 100 & Cameroun & \\
\hline & & & & 90 & Libéria & \\
\hline & & & & 60 & CI & \\
\hline & & & & 80 & $\mathrm{RC}$ & \\
\hline & & & & 80 & $\mathrm{RDC}$ & \\
\hline & & & & 80 & RCA & \\
\hline \multirow[t]{10}{*}{ Tiama blanc } & E. angolense & 55 & & 80 & RCA & Petrucci et al., 1995 \\
\hline & & 50 & & 80 & RCA & Yalibanda, 1999 \\
\hline & & & 85 & & Ouganda & Plumptre, 1995 \\
\hline & & & & 110 & Ghana & Tchinda, 2008 \\
\hline & & & & 90 & Libéria & \\
\hline & & & & 60 & $\mathrm{CI}$ & \\
\hline & & & & 80 & Gabon & \\
\hline & & & & 80 & $\mathrm{RC}$ & \\
\hline & & & & 80 & Cameroun & \\
\hline & & & & 80 & RDC & \\
\hline
\end{tabular}

- : pas d'information - no information; RC : République du Congo; RDC : République Démocratique du Congo ; RCA : République centrafricaine; CI : Côte d'Ivoire. 
globalement une courbe exponentielle décroissante ou une courbe à décroissance linéaire, avec un pic dans les classes intermédiaires où les jeunes tiges sont mieux représentées (généralement dans le sous-bois en attente d'une ouverture du couvert pour pouvoir se développer). La régénération n'est donc pas déficitaire (FRM, 2007 ; FRM, 2009 ; Eckebil et al., 2017 ). Pour stabiliser l'effectif exploitable de cette espèce, il faudrait une limitation des taux de prélèvement ou un allongement de la durée de rotation (Karsenty \& Gourlet-Fleury, 2006 ; Eckebil et al., 2017). La structure des populations de kosipo, sipo et tiama blanc est variable, leurs courbes de distribution ne sont généralement pas caractéristiques. Ainsi, l'exploitation de chacune de ces espèces nécessite une attention particulière. De multiples mesures sont à prendre (recommandations majeures) afin de garantir leur maintien. Parmi ces mesures, on citera notamment l'augmentation du diamètre minimum d'aménagement (DMA) ou la limitation des coefficients d'exploitation, une aide à la régénération et voire même l'interdiction de leur exploitation dans certaines parcelles ou séries d'aménagement (Daïnou \& Doucet, 2010).

\section{CONCLUSIONS ET PERSPECTIVES}

La présente synthèse bibliographique montre que la taxonomie du genre Entandrophragma a subi une évolution importante depuis que ce nom générique a été proposé en 1894 par Casimir de Candolle. Néanmoins, quelques incertitudes demeurent sur la délimitation des espèces, en particulier E. congoense qui a été mis en synonymie avec E. angolense par certains auteurs, alors qu'elle est reconnue comme espèce à part entière par d'autres. Ce problème pourrait être résolu notamment à l'aide d'outils de génétique moléculaire. Les connaissances sur l'écologie et la génétique des espèces d'Entandrophragma demeurent tout aussi insuffisantes et méritent d'être approfondies. Ainsi, les recherches futures pourraient notamment se focaliser sur :

- la mise à jour de la distribution géographique de chaque espèce ;

- la phylogénie moléculaire susceptible de comprendre les patrons de distributions des espèces au sein du genre ;

- la connaissance détaillée de tempérament de chaque espèce par la quantification des diamètres des vaisseaux de la moelle à l'écorce du bois ;

- la connaissance fine de la phénologie de chaque espèce en tenant compte de la diversité des habitats et des variations du climat dans toute l'aire de distribution du genre ;

- l'amélioration des techniques pour favoriser la régénération naturelle ;
- l'amélioration des connaissances sur la dissémination et la fructification des espèces.

Ces nouvelles investigations scientifiques contribueront à l'amélioration de la durabilité des espèces d'Entandrophragma.

\section{Remerciements}

Nous adressons nos remerciements aux projets «FCCC » et «FORETS », financés par l'Union européenne et mis en ouvre à l'Université de Kisangani par le CIFOR en collaboration avec R\&SD, pour l'octroi de la bourse doctorale, au Musée Royal de l'Afrique Centrale pour la bourse de stage scientifique «ABIC » (2016 et 2017), au Centre de Recherche à Yangambi de l'Institut National pour l'Étude et la Recherche Agronomique (INERA) pour l'accès régulier à sa bibliothèque centrale et à Jean-Yves de Vleeschouwer (Laboratoire de Foresterie des Régions tropicales et subtropicales, Université de Liège - Gembloux Agro-Bio Tech) pour la mise à disposition de la documentation pertinente. Qu'ils soient remerciés tous ceux qui nous ont appuyés à la recherche et à la compilation des informations liées à cette publication. Merci aussi à la congrégation des Prêtres du Sacré Cour de Jésus pour l'espace méditatif accordé durant nos divers séjours de travail au Scolasticat Père Déhon à Kisangani et à la Communauté de Lemba à Kinshasa (RDC).

\section{Bibliographie}

Beeckman H., 2016. Wood anatomy and trait-based ecology. IAWA J., 37, 127-151.

Biwolé A.B., Bourland N., Daïnou K. \& Doucet J.-L., 2012. Définition du profil écologique de l'azobé, Lophira alata, une espèce ligneuse africaine de grande importance : synthèse bibliographique et perspectives pour des recherches futures. Biotechnol. Agron. Soc. Environ., 16, 217-228.

Burrows J., Schmidt E., Burrows S. \& Lötter M., 2017. Trees \& shrubs of Mozambique. Johannesburg, South Africa: Jacana Publishers.

Chevallier M.-H. et al., 2010. Les genres Entandrophragma (Meliaceae) et Dacryodes (Burseraceae) en Afrique centrale atlantique. In : Actes du colloque final de restitution du projet IFORA, Agropolis International, 21-22 juin 2010, Montpellier, France.

CJBG (Conservatoire et Jardin botaniques de la Ville de Genève), 2012. Base de données des plantes d'Afrique. Genève, Suisse : CJBG.

Daïnou K. \& Doucet J.-L., 2010. Évaluation de l'aménagement et de l'état des populations des essences exploitées par le Groupe SEFAC. Wavre, Belgique : Nature + .

de Candolle C., 1894. Meliaceae Novae. Bull. Herb. Boissier, II(9), 583-584. 
de la Mensbruge G., 1966. La germination et les plantules des essences arborées de la forêt dense humide de la Côte d'Ivoire. Nogent-sur-Marne, France : Centre Technique Forestier Tropical.

de Wilde H.J.E., 2015. Meliaceae. In : Sosef S.M. Florence J., Banak L.N. \& Bourobou H.P., éds. Flore du Gabon. 47 : Aloaceae, Meliaceae, Rhizophoraceae. Leiden, Germany : Margraf Publishers, Weikersheim Backhuys Publishers.

Dikumbwa N., 1990. Facteurs écoclimatiques et sur les cycles biogéochimiques en forêt dense sèche zambézienne (Muhulu) du Shaba méridional. Géo-ÉcoTrop, 14, 1-159.

Doucet J.-L., 2003. L'alliance délicate de la gestion forestière et de la biodiversité dans les forêts du centre $d u$ Gabon. Thèse de doctorat : Faculté universitaire des Sciences agronomiques de Gembloux (Belgique).

Doucet J.-L. et al., 2016. Enrichment of Central African logged forests with high-value tree species: testing a new approach to regenerating degraded forests. Int. J. Biodivers. Sci. Ecosyst. Serv. Manage., 12(1-2), 8395.

Doumenge C. et al., 2010. Évaluation des niches climatiques de quatre espèces d'Entandrophragma (Meliaceae) d'Afrique occidentale et centrale. Implications évolutives et paléoclimatiques. In : Actes du $19^{e}$ Congrès AETFAT, 25-30 avril 2010, Madagascar. Meise, Belgique : National Botanic Garden of Belgium.

Durrieu de Madron L., Favrichon V. \& Dupuy B., 1998. Croissance et productivité en forêt dense humide : bilan des expérimentations dans le dispositif de Mopri-Côte d'Ivoire (1978-1992). Montpellier, France : Cirad-Forêt.

Durrieu de Madron L. \& Daumerie A., 2004. Diamètre de fructification de quelques essences en forêt naturelle centrafricaine. Bois For. Trop., 281(3), 87-95.

Eckebil P.P.T. et al., 2017. Entandrophragma cylindricum (Sprague) Sprague (Meliaceae), une espèce ligneuse concurrentielle en Afrique centrale (synthèse bibliographique). Biotechnol. Agron. Soc. Environ., 21, 80-97.

FAO, 2011. La situation des forêts dans le bassin amazonien, le bassin du Congo et l'Asie du Sud-Est. Rapport préparé pour le sommet des trois bassins forestiers tropicaux du 31 mai au 3 juin 2011 à Brazzaville, République du Congo. Rome : FAO, OIBT.

Fayolle A. et al., 2015. Differential performance between two timber species in forest logging gaps and in plantations in Central Africa. Forests, 6, 380-394.

FRM (Forêt Ressources Management), 2007. Plan d'aménagement des unités forestières : 09-003,09-004a, 09-005a et 09-005b-SFID. Montpellier, France : FRM.

FRM (Forêt Ressources Management), 2009. Plan d'aménagement des unités forestières : 09-007 et 09-008 -SFID. Montpellier, France : FRM

Garcia F., Noyer J.-L., Risterucci A.-M. \& Chevallier M.-H., 2004. Genotyping of mature trees of Entandrophragma cylindricum with microsatellites. J. Heredity, 95, 454457.

Gillet J.-F., Ngalouo B. \& Missamba-Lola A.P., 2008. Rapport d'analyse - volet dynamique forestière. Projet CIB FFEM «Suivi du programme dynamique forestièreagroforesterie - inventaires faunes ». Brazzaville, République du Congo : Ministère de l'Économie Forestière.

Hall J.S., 2008. Seed and seedling survival of African mahogany (Entandrophragma spp.) in the Central African Republic: implications for forest management. For. Ecol. Manage., 255, 292-299.

Hall J.S., 2011. Natural forest silviculture for Central African Meliaceae. In: Günter S., Weber M., Stimm B. \& Mosandl R., eds. Silviculture in the Tropics. Berlin: Springer.

Hall J.S., Harris D.J., Medjibe V. \& Ashton P.M.S., 2003. The effects of selective logging on forest structure and tree species composition in a Central African forest: implications for management of conservation areas. For. Ecol. Manage., 183, 249-264.

Hall J.S., McKenna J.J., Ashton P.M.S. \& Gregoire T.G., 2004. Habitat characterizations understimate the role of edaphic factors controlling the distribution of Entandrophragma. For. Ecol. Manage., 85, 2171-2183.

Jolivet C. \& Degen B., 2012. Use of DNA fingerprints to control the origin of sapelli timber (Entandrophragma cylindricum) at the forest concession level in Cameroon. Forensic Sci. Int., 6, 487-493.

Karsenty A. \& Gourlet-Fleury S., 2006. Assessing sustainability of logging practices in the Congo Basin's managed forests: the issue of commercial species recovery. Ecol. Soc., 11, 26.

Kenfack D., 2011. Resurrection in Carapa (Meliaceae): a reassessment of morphological variation and species boundaries using multivariate methods in a phylogenetic context. Bot. J. Linn. Soc., 165, 186-221.

Koenen E.J., Clarkson J.J., Pennington T.D. \& Chatrou L.W., 2015. Recently evolved diversity and convergent radiations of rainforest mahoganies (Meliaceae) shed new light on the origins of rainforest hyperdiversity. New Phytol., 207(2), 327-339.

Lemmens R.H.M.J., 2008. Entandrophragma excelsum (Dawe \& Sprague) Sprague. In: Louppe D., OtengAmoako A.A. \& Brink M., eds. Prota 7(1): Timbers/Bois d'auvre 1. Wageningen, The Netherlands: Prota.

Louis J. \& Fouarge J., 1947. Essences forestières et bois du Congo. 4 : Entandrophragma palustre. Bruxelles : Institut National pour l'Étude Agronomique du Congo belge (INEAC).

Lourmas M. et al., 2007. Reduced density due to logging and its consequences on mating system and pollen flow in the African mahogany Entandrophragma cylindricum. Heredity, 99, 151-160.

Makana J.-R. \& Thomas S.C., 2004. Dispersal limits natural recruitment of African mahoganies. Oikos, 106, 67-72. 
Malaisse F., 1993. The ecology of the Zambezian dry evergreen forest with recommendations for conservation management. In: Lieth H. \& Lohmann M., eds. Restoration of tropical forest ecosystems. Dordrecht, The Netherlands: Kluwer Academic Press, 75-90.

Malaisse F., Dikumbwa N'L., Blancke V. \& Leteinturier B., 2000. Preliminary observations on Zambezian dry evergreen forest gaps. In: Ceulemans R., Bogaert J., Deckmyn G. \& Nijs I., eds. Topics in ecology: structure and function in plants and ecosystem. Antwerp, Belgium: University of Antwerp, 143-153.

Mangenot S. \& Mangenot G., 1962. Enquête sur les nombres chromosomiques dans une collection d'espèces tropicales. Bull. Soc. Bot. Fr., 109, 411-447.

Medjibe V. \& Hall J.S., 2002. Seed dispersal and its implications for silviculture of African mahogony (Entandrophragma spp.) in undisturbed forest in the Central African Republic. For. Ecol. Manage., 170, 249257.

Menga P., Bayol N., Nasi R. \& Fayolle A., 2012. Phénologie et diamètre de fructification du wengé, Millettia laurentii De Wild. : implications pour la gestion. Bois For. Trop., 312(2), 31-41.

Meunier Q., Moumbogou C. \& Doucet J.-L., 2015. Les arbres utiles du Gabon. Gembloux, Belgique : Les Presses agronomiques de Gembloux.

Monthe F. et al., 2017a. Extensive seed and pollen dispersal and assortative mating in the rain forest tree Entandrophragma cylindricum (Meliaceae) inferred from indirect and direct analyses. Mol. Ecol., 26(19), 5279.

Monthe F. et al., 2017b. Characterization of microsatellite markers in two African exploited trees, Entandrophragma candollei and E. utile (Meliaceae). Appl. Plant Sci., 5, 1600130.

Muellner A.N., Savolainen V., Samuel R. \& Chase M.W., 2006. The mahogany family "out-of-Africa": divergence time estimation, global biogeographic patterns inferred from plastid rbcL DNA sequences, extant, and fossil distribution of diversity. Mol. Phylogenet. Evol., 40, 236250 .

Muellner A.N., Pennington T.D. \& Chase M.W., 2009. Molecular phylogenetics of neotropical Cedreleae (mahogany family, Meliaceae) based on nuclear and plastid DNA sequences reveal multiple origins of "Cedrela odorata". Mol. Phylogenet. Evol., 52, 461469.

Muellner A.N., Schaefer H. \& Lahaye R., 2011. Evaluation of candidate DNA barcoding loci for economically important timber species of the mahogany family (Meliaceae). Mol. Phylogenet. Evol., 11, 450-460.

Mujuni D.B., 2008. Entandrophragma utile (Dawe \& Sprague) Sprague. In: Louppe D., Oteng-Amoako A.A. \& Brink M., eds. Prota 7(1): Timbers/Bois d'auvre 1. Wageningen, The Netherlands: Prota.

Nature +, 2011. Révision de plan d'aménagement : unité forestière d'aménagement $n^{\circ} 09-021$ (Province du Sud-
Arrondissement de Ma'an). Douala, Cameroun : Wijma Douala.

Nyunaï N., 2008. Entandrophragma candollei Harms. In: Louppe D., Oteng-Amoako A.A. \& Brink M., eds. Prota 7(1): Timbers/Bois d'œuvre 1. Wageningen, The Netherlands: Prota.

Onguene N.A. \& Kuyper T.W., 2001. Mycorrhizal associations in the rain forest of South Cameroon. For. Ecol. Manage., 140, 277-287.

Owona Ndongo P.-A. et al., 2009. Plantations de bois d'œuvre en zone équatoriale africaine : cas de l'arboretum de l'Enef de Mbalmayo au sud du Cameroun. Bois For. Trop., 299, 37-48.

Palla F., Louppe D. \& Forni E., 2002. Sapelli. Fiche technique, écologique et sylvicole. Montpellier, France : Cirad-Forêt.

Pennington T.D., Muellner A.N. \& Wise R., 2010. A monograph of Cedrela (Meliaceae). Milborne Port, UK: Dh Books.

Petrucci Y., Tandeau de Marsac G. \& Morel P.J., 1995. Évolution du peuplement adulte et de la régénération acquise après interventions sylvicoles. Dispositif de recherche en forêt dense de Boukoko-La Lolé. Appui à la recherche forestière Fac/Arf. Bangui, République centrafricaine : Ministère des Eaux, Forêts, Chasse et Pêche.

Plumptre A.J., 1995. The importance of "seed trees" for the natural regeneration of selectively logged tropical forest. Common For. Rev., 74, 253-258.

Poorter L., Bongers F., Kouamé F.Y.N. \& Hawthorne W.-D., 2004. Biodiversity of West African forests. An ecological atlas of woody plant species. Wallingford, UK: Cabi.

Roux J.P., 2003. Flora of South Africa. Cape Town: South African National Biodiversity Institute.

Sépulchre F., Daïnou K. \& Doucet J.-L., 2008. Étude de la vulnérabilité de 18 essences ligneuses commerciales d'Afrique centrale reprises sur la liste rouge de l'UICN. Gembloux, Belgique : Faculté universitaire des Sciences Agronomiques de Gembloux.

Staner P., 1943. Les acajous du Congo Belge. Bull. Agric. Congo Belg., 34(1-2), 163-246.

Tailfer Y., 1989. La forêt dense d'Afrique Centrale. Identification pratique des principaux arbres. I. Approche forestière et morphologique. Wageningen, Pays-Bas : CTA.

Taylor C.J., 1960. Synecology and silviculture in Ghana. London: Thomas Nelson and Co.

Tchinda A.T., 2008. Entandrophragma angolense (Welw.) C.DC. In: Louppe D., Oteng-Amoako A.A. \& Brink M., eds. Prota 7(1): Timbers/Bois d'œuvre 1. Wageningen, The Netherlands: Prota.

The Plant List, 2013. Version 1.1, http://www.theplantlist. org/tpl1.1/search?q=Entandrophragma (27 February 2018).

Voorhoeve A.G., 1965. Liberian high forest trees. A systematical botanical study of the 75 most important 
or frequent high forest trees, with reference to numerous related species. Wageningen, The Netherlands: Centre for Agricultural Publication and Documentation.

Welwitsch F., 1858. Apontamentos Phyto-Geographicos. Ann. Conselho Ultramarino, parte nâo official, Lisbonne, I, 526-580.

White F., 1986. La végétation de l'Afrique. Mémoire accompagnant la carte de végétation de l'Afrique. Paris : Orstom et Unesco.

Wilks C. \& Issembé Y., 2000. Guide pratique d'identification : les arbres de la Guinée Équatoriale, région continentale. Wetter, Allemagne : Curef.
Yalibanda Y., 1999. Phénologie en forêt dense de Ngotto (Rca). Bilan de trois années d'observation. In : Nasi R., Amsallem I. \& Drouineau S., éds. La gestion des forêts denses africaines aujourd'hui. Actes du séminaire Forafri de Libreville (Gabon), 12-16 octobre 1998, Montpellier, France. Montpellier, Cirad : France.

(67 réf). 